\title{
Determinación del nivel de desempeño de un edificio habitacional estructurado en base a muros de hormigón armado y diseñado según normativa chilena
}

\author{
Determination of the performance level of a residential building based on reinforced concrete \\ walls and designed according to Chilean codes \\ Fecha de entrega: 3 de julio 2017 \\ Fecha de aceptación: 16 de abril 2018
}

\section{Ricardo Medina y Juan Music}

Departamento de Ingeniería Civil, Universidad Católica del Norte, Av. Angamos 0610, Antofagasta, Chile,

rmedinaf.contacto@gmail.com,jmusic@ucn.cl

Se determina el nivel de desempeño de un edificio estructurado en base a muros de hormigón armado, el cual es diseñado según la normativa chilena vigente (DS60, 2011, DS61, 2011). Para determinar el nivel de desempeño se utiliza el Método de Espectro de Capacidad MEC, el cual superpone el espectro de capacidad y demanda sísmica. El espectro de capacidad se construye a partir de la curva de capacidad estructural del edificio, la cual se obtiene mediante un análisis estático no lineal pushover. Al utilizar esta herramienta se deben incorporar propiedades no lineales a los materiales que componen los muros usando leyes constitutivas (relaciones tensión-deformación). Para el análisis pushover se consideran 3 patrones de carga. Los espectros de demanda sísmica considerados se obtienen según movimientos sísmicos de diseño establecidos por Lagos et al. (2012) provenientes de estudios de riesgo sísmico en edificios chilenos. Además, se considera dos formas para la determinación del espectro de desplazamiento elástico: la establecida en el DS61 (2011) y la señalada por ATC y FEMA. A partir de los puntos de desempeño obtenidos se determinan distintos parámetros, tales como: desplazamientos y cortes por piso y tensiones en los muros. Con el fin de comparar los resultados obtenidos con lo propuesto por Vision 2000, se determina el desplazamiento objetivo (cociente entre desplazamiento de techo y altura del edificio). Se concluye que el edificio analizado cumple, para todos los puntos de desempeño determinados, que el desplazamiento objetivo no supera los límites establecidos por Visión 2000. Finalmente, el nivel de desempeño del edificio, para todos los casos considerados, no supera el operacional, lo cual coincide con la experiencia chilena, según Lagos et al. (2012).

Palabras clave: nivel de desempeño, Vision 2000, método espectro de capacidad, elemento shell multicapa
The performance level of a residential building based on reinforced concrete walls was determined. The building was designed according with the current Chilean regulations (DS60, 2011, DS61, 2011). To determine the performance level, the Capacity Spectrum Method CSM was used, which superimposes the capacity and the seismic demand spectra. Capacity spectrum is built from the structural capacity curve of the building, which is obtained by performing a nonlinear static analysis (pushover). To use this tool, nonlinear properties of the materials must be incorporated in the wall elements using constitutive laws (stress-strain relationships). For the pushover analysis, three load patterns were considered. The seismic demand spectra considered in this study was obtained from design seismic movements proposed by Lagos et al. (2012) based on studies of seismic risk in Chilean buildings. Also two ways for the determination of the elastic displacement spectrum were considered: the one provided by the DS61 (2011) and that provided by ATC and FEMA. Different parameters such as displacement and shear by story and stresses in the walls were determined from the performance points obtained. In order to compare the results obtained in this research with those proposed by Vision 2000, the target displacement (ratio between roof displacement and height of the building) was calculated. As a conclusion, for all determined performance points of the building, the objective displacement does not exceed the limits established by Vision 2000. Also the performance level of the building, for all cases considered, does not exceed the operational level, which coincides with the Chilean experience, according to Lagos et al. (2012).

Keywords: performance level, Vision 2000, capacity spectrum method, multilayer shell element 


\section{Introducción}

La comunidad internacional de Ingeniería Estructural ha resaltado la importancia de complementar la fase numérica del diseño sísmico con una fase conceptual y de implementación, basada en el control de la respuesta dinámica de las estructuras sismorresistentes. En este contexto, la Sociedad de Ingenieros Estructurales de California SEAOC estableció el Comité Visión 2000 (1995). Este Comité describe el concepto de diseño por desempeño como la selección de los objetivos de diseño, sistemas estructurales y configuración apropiados (fase conceptual), el dimensionamiento y detallado de una estructura (fase numérica) y la garantía del control de la calidad durante la construcción y del mantenimiento a largo plazo.

La Ingeniería Sísmica basada en el desempeño busca como objetivo que los daños esperados estén dentro de márgenes preestablecidos para diferentes sismos de diseño con un aceptable nivel de confiabilidad. Las variables con que se cuentan para lograrlo son la rigidez, resistencia y capacidad de incursionar fuera del rango lineal. Los avances logrados han permitido plantear requerimientos de diseño sísmico basados en esta filosofía y sugieren que la siguiente generación de códigos estará basada en ella.

El Método de Espectro de Capacidad MEC, ha sido incluido por ATC-40 (1996) y en diversos documentos por FEMA 273 (1997), FEMA 274 (1997), FEMA 356 (2000) y FEMA 440 (2005). Este método compara la capacidad de una estructura para resistir fuerzas laterales con la demanda sísmica, representadas a través de diagramas espectrales. La superposición de la capacidad de la estructura con la demanda sísmica da origen al punto de desempeño.

La filosofía de diseño sísmico chilena, al igual que en el resto del mundo establece como prioridad la seguridad de la vida y el no colapso de una estructura durante un sismo de gran intensidad. En la práctica, en Chile el diseño por desempeño utilizando el MEC, en términos generales, es actualmente usado en algunas oficinas de ingeniería cuando involucran un diseño especial o complejo en edificios de gran altura. Este tipo de análisis se ha realizado en nuestro país en edificios de gran altura de hormigón armado tales como el Territoria 3000, Torre Telefónica y Torre 2 de Costanera Center, evaluando el comportamiento ante el sismo ocurrido el 27 de febrero de 2010 (Lagos et al., 2012).

Debido al reciente cambio en la normativa de diseño de muros de hormigón armado en Chile, existe un interés en evaluar el nivel de desempeño en edificios de altura. Es por esto que la evaluación por desempeño podría contribuir en un futuro a complementar la normativa chilena vigente. El objetivo general de este estudio es determinar el nivel de desempeño que alcanza un edificio estructurado en base a muros de hormigón armado. Dichos muros son diseñados de acuerdo a la normativa chilena vigente (DS60, 2011; DS61, 2011).

\section{Descripción del edificio analizado}

El edificio analizado se encuentra estructurado en base a muros de hormigón armado de 20 a $40 \mathrm{~cm}$ de espesor conectados mediante vigas de $50 \mathrm{~cm}$ de alto. El espesor de los muros varía cada 5 pisos (subterráneo, 1 a 5, 6 a 10,11 a 15,16 a 20) y mantienen la misma distribución para evitar discontinuidades o singularidades en los muros principalmente a nivel de subterráneo. El edificio cuenta con 20 pisos más 2 subterráneos, contabilizando 22 plantas, cada una de $256 \mathrm{~m}^{2}$. La densidad de muros promedio por pisos es de $2.8 \%$ en $X$ y $3.5 \%$ en $Y$. Todos los elementos estructurales son de hormigón armado G30 (H-35) y acero de calidad A630-420H. Se considera que el edificio se encuentra en zona sísmica 3 y está fundado en suelo tipo B (DS61, 2011). En la Figura 1 se muestra una vista en planta y en 3D del edificio modelado con el software ETABS (2015), en donde se etiqueta cada muro con un número y forma (L, I, T, C).

\section{Análisis sísmico}

El análisis sísmico, se realizó mediante el software ETABS (2015) y considerando la normativa vigente en nuestro país, es decir, aplicando el DS61 (2011) y NCh433 (2010). Las combinaciones de carga consideradas en el análisis del edificio, corresponden a las de un análisis dinámico de superposición modal espectral, establecidos en la norma NCh3171 (2010), en donde la torsión accidental se considera desplazando la ubicación del centro de masa en un 5\%, para cada una de las direcciones de análisis. Los estados de cargas considerados e ingresados al software ETABS se señalan en la Tabla 1. 
a)

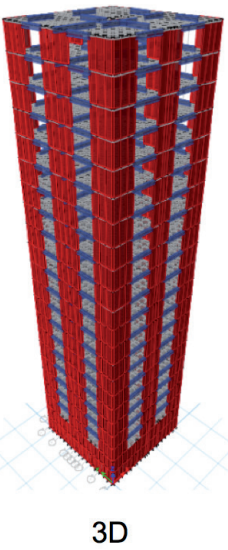

b)

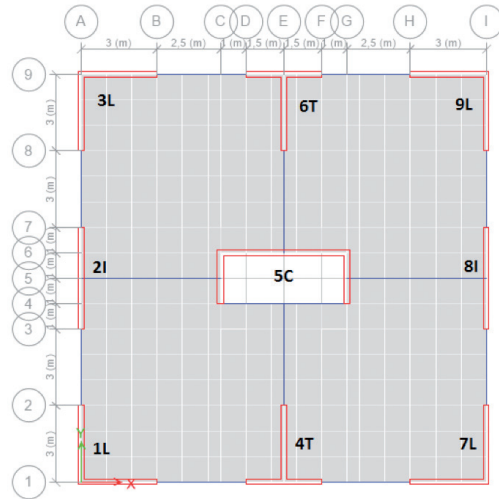

Planta pisos 1 a 20

c)

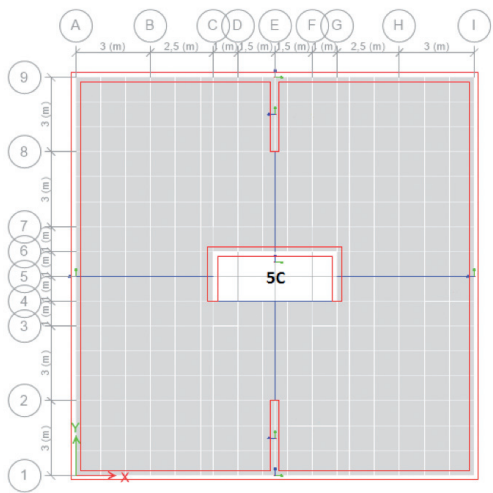

Planta subterráneos -1 y -2

Figura 1: a) Vista tridimensional del modelo, b) y c) plantas del edificio analizado

Tabla 1: Combinaciones de carga ingresadas al software ETABS

\begin{tabular}{|c|c|c|c|}
\hline $\begin{array}{c}\text { Nombre } \\
\text { entrada } \\
\text { ETABS }\end{array}$ & $\begin{array}{c}\text { Estados de carga } \\
\text { según NCh3171 } \\
(2010)\end{array}$ & $\begin{array}{c}\text { Nombre } \\
\text { entrada } \\
\text { ETABS }\end{array}$ & $\begin{array}{c}\text { Estados de carga } \\
\text { según NCh3171 } \\
(2010)\end{array}$ \\
\hline $\mathrm{C} 1$ & $1.4 \mathrm{PP}$ & $\mathrm{C} 6$ & $0.9 \mathrm{PP} \pm 1.4 \mathrm{SY}$ \\
\hline $\mathrm{C} 2$ & $1.2 \mathrm{PP}+1.6 \mathrm{SC}$ & $\mathrm{C} 7$ & $1.2 \mathrm{PP}+\mathrm{SC} \pm 1.4 \mathrm{SEX}$ \\
\hline $\mathrm{C} 3$ & $1.2 \mathrm{PP}+\mathrm{SC} \pm 1.4 \mathrm{SX}$ & $\mathrm{C} 8$ & $0.9 \mathrm{PP} \pm 1.4 \mathrm{SEX}$ \\
\hline $\mathrm{C} 4$ & $0.9 \mathrm{PP} \pm 1.4 \mathrm{SX}$ & $\mathrm{C} 9$ & $1.2 \mathrm{PP}+\mathrm{SC} \pm 1.4 \mathrm{SEY}$ \\
\hline $\mathrm{C} 5$ & $1.2 \mathrm{PP}+\mathrm{SC} \pm 1.4 \mathrm{SY}$ & $\mathrm{C} 10$ & $0.9 \mathrm{PP} \pm 1.4 \mathrm{SEY}$ \\
\hline
\end{tabular}

En la Tabla 2 se muestran los resultados de periodos y masas equivalentes, en la Figura 2 se muestran los espectros elásticos y de diseño y en la Figura 3 se muestra la verificación de desplazamientos.

Tabla 2: Periodos y porcentaje de masa equivalente asociada a cada modo

\begin{tabular}{|c|c|c|c|c|c|c|c|}
\hline \multirow{2}{*}{ Modo } & \multirow{2}{*}{$\begin{array}{c}\text { Periodo } \\
T, \mathrm{~s}\end{array}$} & \multicolumn{3}{|c|}{$\begin{array}{c}\text { \% } \\
\text { libertán grado de }\end{array}$} & \multicolumn{3}{|c|}{$\%$ acumulado } \\
\cline { 3 - 9 } & & UX & UY & RZ & SumUX & SumUY & SumRZ \\
\hline 1 & 1.258 & 63.21 & 0 & 0.05 & 63.21 & 0 & 0.05 \\
\hline 2 & 1.205 & 0 & 63.87 & 0 & 63,21 & 63.87 & 0.05 \\
\hline 3 & 0.745 & 0.03 & 0 & 63.77 & 63.24 & 63.87 & 63.82 \\
\hline 4 & 0.350 & 13.47 & 0 & 0.01 & 76.71 & 63.87 & 63.83 \\
\hline 5 & 0.348 & 0 & 12.75 & 0 & 76.71 & 76.63 & 63.83 \\
\hline 6 & 0.227 & 0.03 & 0 & 11.31 & 76.75 & 76.63 & 75.14 \\
\hline 7 & 0.170 & 0 & 5.71 & 0 & 76.75 & 82.34 & 75.14 \\
\hline 8 & 0.166 & 5.95 & 0 & 0.02 & 82.69 & 82.34 & 75.16 \\
\hline 9 & 0.115 & 0.05 & 0 & 5.62 & 82.75 & 82.34 & 80.78 \\
\hline 10 & 0.101 & 0 & 3.75 & 0 & 82.75 & 86.09 & 80.78 \\
\hline 11 & 0.099 & 3.69 & 0 & 0.04 & 86.44 & 86.09 & 80.82 \\
\hline 12 & 0.069 & 0.13 & 0 & 3.65 & 86.56 & 86.09 & 84.47 \\
\hline 13 & 0.068 & 0 & 3.00 & 0 & 86.56 & 89.09 & 84.47 \\
\hline 14 & 0.067 & 2.66 & 0 & 0.12 & 89.22 & 89.09 & 84.59 \\
\hline 15 & 0.050 & 0 & 2.58 & 0 & 89.22 & 91.66 & 84.59 \\
\hline 16 & 0.050 & 2.37 & 0 & $2.96 \mathrm{E}-4$ & 91.59 & 91.66 & 84.59 \\
\hline
\end{tabular}

a)

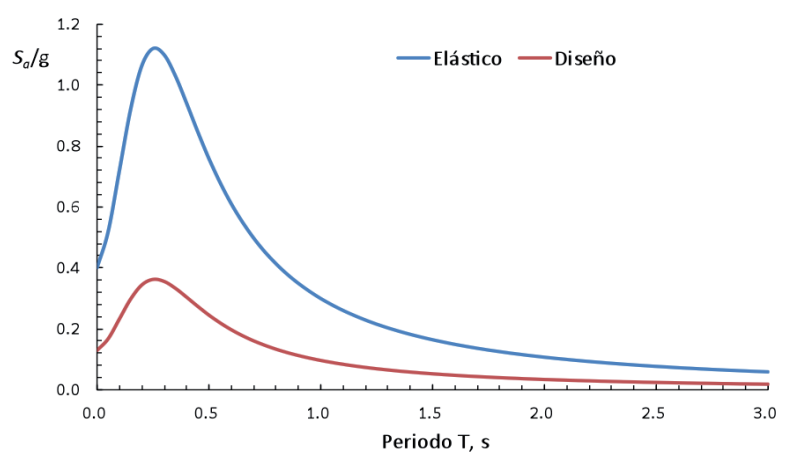

b)

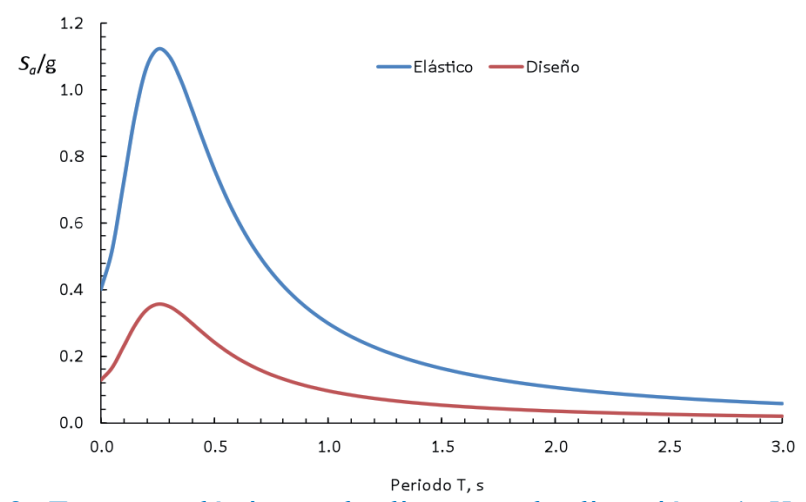

Figura 2: Espectro elástico y de diseño en la dirección: a) $X$, $\left.R^{*}{ }_{\text {modif }}=3.11 \mathrm{y} \mathrm{b}\right) Y, R^{*}{ }_{\text {modif }}=3.14$ 
a)

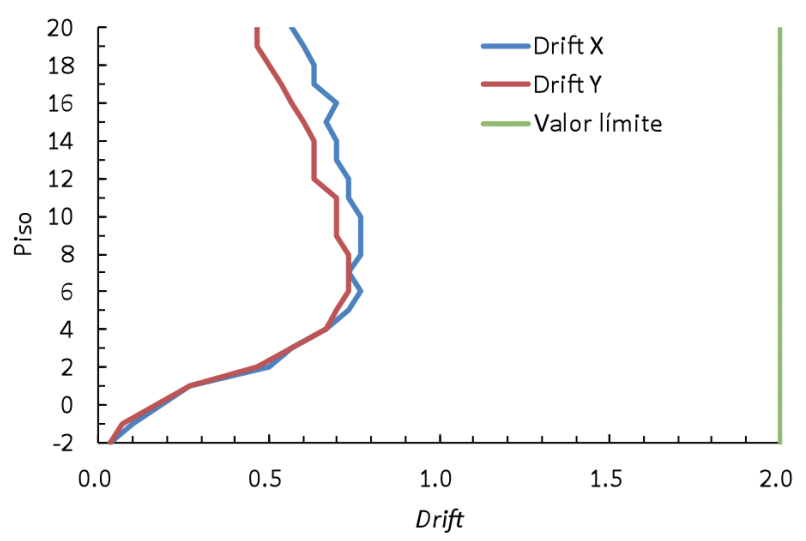

b)

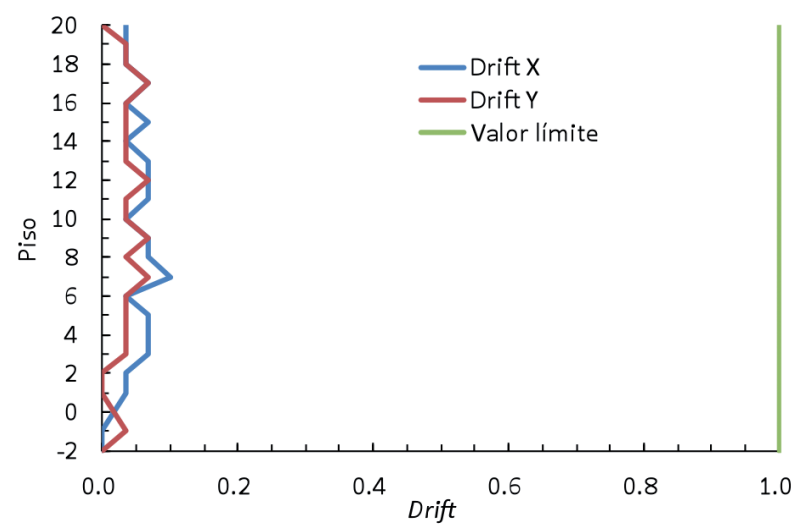

Figura 3: Verificación de control de desplazamientos de entrepisos: a) del centro de masa y b) diferencia entre centro de masa y un punto extremo

\section{Díseño de muros}

Los muros de hormigón armado han sido diseñados según los requerimientos del DS60 (2011). En la Figura 4 se muestra el diseño y armado de los tipos de muros que componen el edificio.

\section{Obtención de la capacidad del edificio}

La capacidad del edificio se representa mediante curvas de capacidad. Estas curvas se obtienen mediante un análisis estático no lineal pushover. Este análisis se realiza con la ayuda del software ETABS utilizando una modelación shell multicapa para los muros y frame para las vigas considerando para estas un comportamiento lineal. Se consideran tres patrones de carga.

\section{Modelo shell multicapa}

Los modelos shell multicapa están basados principalmente en el método de elementos finitos, usan una alta discretización de cada miembro para lograr representar detalladamente cada material, incluso cada barra de acero de refuerzo. Adicionalmente, el comportamiento de cada material es representado por leyes constitutivas que generalmente son bien conocidas. De esta manera, cualquier configuración del hormigón y del acero de refuerzo puede ser representada (Fahjan et al., 2010). Para modelar los muros utilizando shell multicapa en ETABS, se deben ingresar curvas tensión-deformación que representen el comportamiento no lineal de los materiales que lo componen (leyes constitutivas). A continuación, se describen dichas curvas para cada material:

a) Acero de refuerzo: se utiliza el modelo de Park, este modelo se encuentra incluido en ETABS (2015), debido a esto solo es necesario ingresar parámetros de tensiones de fluencia y ruptura, para una calidad de acero A630-420H. Ver Figura 5a.

b) Hormigón no confinado: se utilizan ecuaciones del modelo de Mander (1988), este modelo se encuentra incluido en ETABS (2015), por lo que solo es necesario ingresar la resistencia del hormigón utilizado G30 (H35). Ver Figura 5b.

c) Hormigón confinado: se utilizan ecuaciones del modelo de Mander (1988), sin embargo, esta curva no se encuentra incluida en ETABS por lo que cada valor debe ser calculado e ingresado manualmente, según el nivel de confinamiento aportado por los estribos. Ver Figura 5b.

\section{Patrones de carga}

Para la realización del análisis pushover y posterior obtención de las curvas de capacidad se utilizaron 3 patrones de carga:

a) Patrón modal: patrón proporcional al producto del modo de vibrar del primer modo de cada dirección $(X$ $Y)$ multiplicada por la masa sísmica en cada piso.

b) Patrón de masas: patrón proporcional a la masa sísmica de cada piso.

c) Patrón triangular invertido: distribución de fuerza lateral se incrementa en una proporción lineal con respecto a la altura desde la base.

En la Figura 6 se comparan los coeficientes de fuerza lateral (proporción de carga en cada piso) de cada patrón para la dirección $X$. Se observa que el patrón modal y el 
Muro tipo L

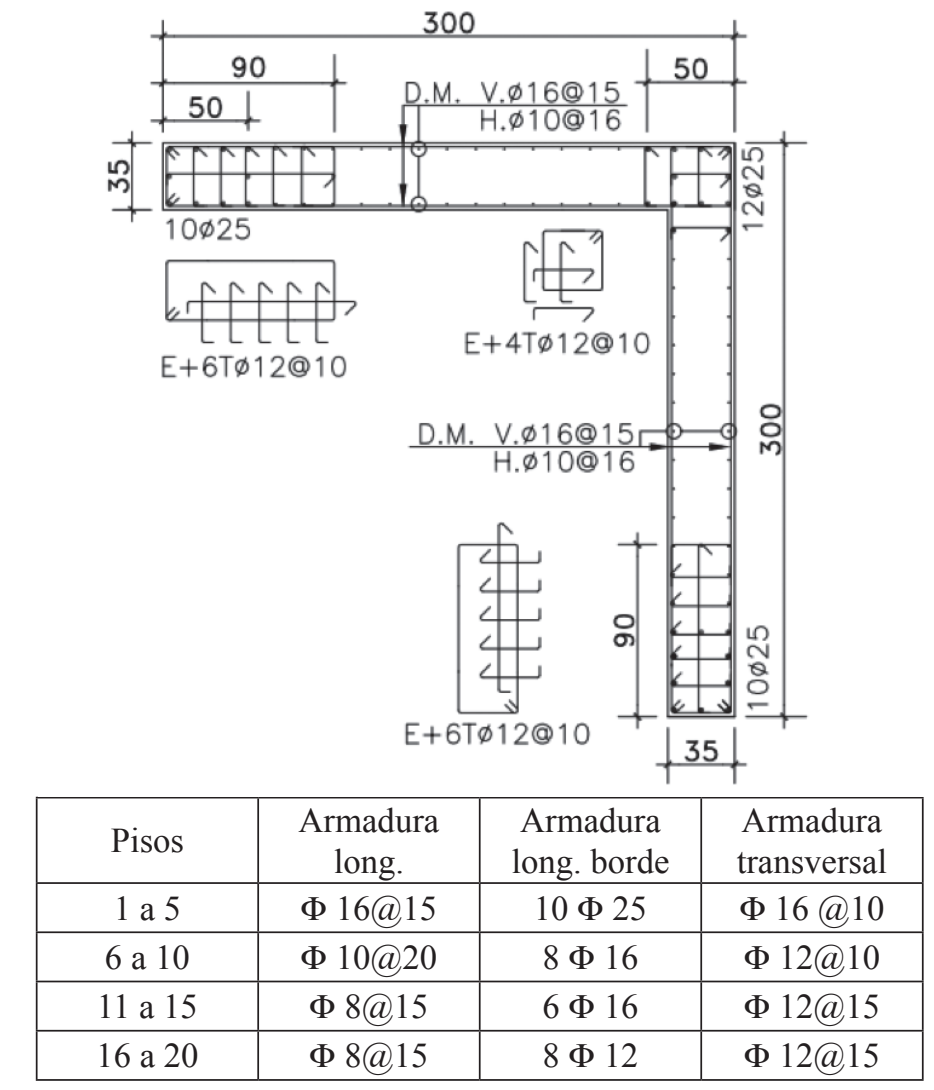

300

Muro tipo T

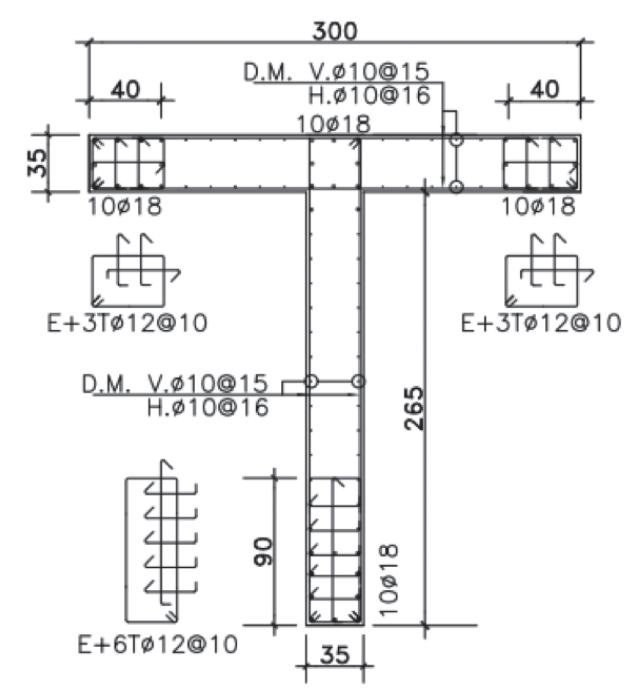

\begin{tabular}{|c|c|c|c|}
\hline Pisos & $\begin{array}{c}\text { Armadura } \\
\text { long. }\end{array}$ & $\begin{array}{c}\text { Armadura } \\
\text { long. borde }\end{array}$ & $\begin{array}{c}\text { Armadura } \\
\text { transversal }\end{array}$ \\
\hline 1 a 5 & $\Phi 10 @ 15$ & $10 \Phi 18$ & $\Phi 16 @ 10$ \\
\hline 6 a 10 & $\Phi 10 @ 20$ & $8 \Phi 16$ & $\Phi 12 @ 10$ \\
\hline 11 a 15 & $\Phi 8 @ 15$ & $6 \Phi 16$ & $\Phi 12 @ 15$ \\
\hline 16 a 20 & $\Phi 8 @ 20$ & $6 \Phi 12$ & $\Phi 12 @ 15$ \\
\hline
\end{tabular}

Figura 4: Diseño y armado de muros tipo L, I, T y C
Muro tipo I

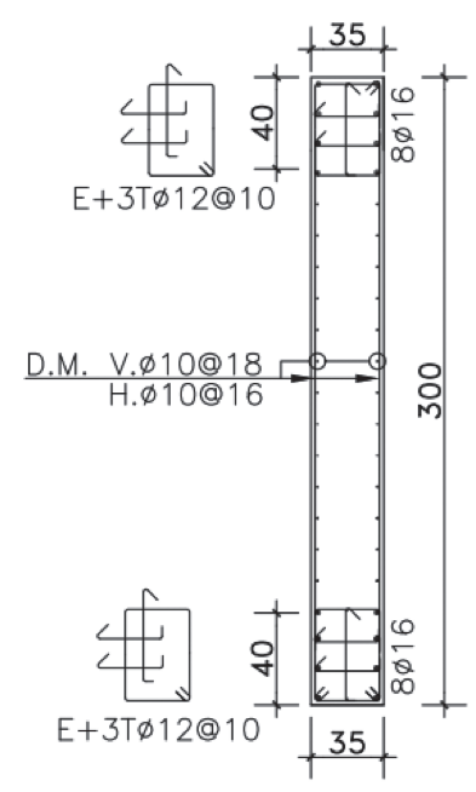

\begin{tabular}{|c|c|c|c|}
\hline Pisos & $\begin{array}{c}\text { Armadura } \\
\text { long. }\end{array}$ & $\begin{array}{c}\text { Armadura } \\
\text { long. borde }\end{array}$ & $\begin{array}{c}\text { Armadura } \\
\text { transversal }\end{array}$ \\
\hline 1 a 5 & $\Phi 10 @ 18$ & $8 \Phi 16$ & $\Phi 12 @ 15$ \\
\hline 6 a 10 & $\Phi 10 @ 20$ & $8 \Phi 16$ & $\Phi 10 @ 20$ \\
\hline 11 a 15 & $\Phi 8 @ 15$ & $8 \Phi 16$ & $\Phi 8 @ 15$ \\
\hline 16 a 20 & $\Phi 8 @ 15$ & $8 \Phi 16$ & $\Phi 8 @ 20$ \\
\hline
\end{tabular}

Muro tipo C

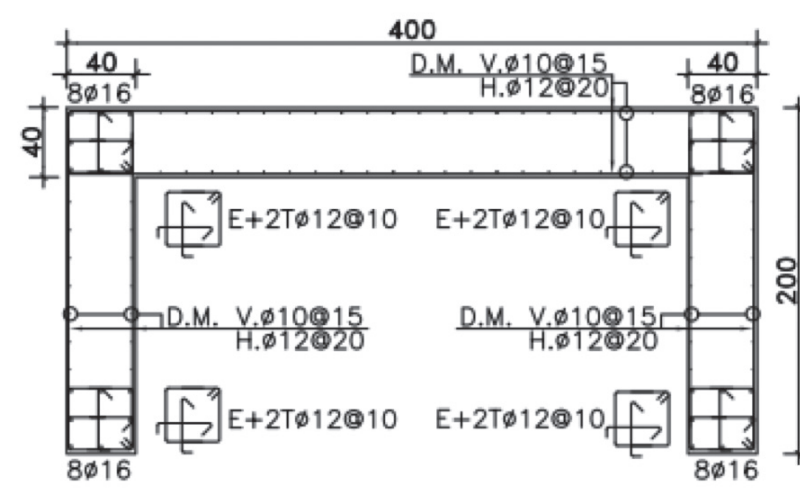

\begin{tabular}{|c|c|c|c|}
\hline Pisos & $\begin{array}{c}\text { Armadura } \\
\text { long. }\end{array}$ & $\begin{array}{c}\text { Armadura } \\
\text { long. borde }\end{array}$ & $\begin{array}{c}\text { Armadura } \\
\text { transversal }\end{array}$ \\
\hline 1 a 5 & $\Phi 10 @ 15$ & $8 \Phi 16$ & $\Phi 18 @ 12$ \\
\hline 6 a 10 & $\Phi 10 @ 20$ & $8 \Phi 16$ & $\Phi 16 @ 12$ \\
\hline 11 a 15 & $\Phi 8 @ 15$ & $6 \Phi 16$ & $\Phi 12 @ 10$ \\
\hline 16 a 20 & $\Phi 8 @ 20$ & $8 \Phi 12$ & $\Phi 12 @ 10$ \\
\hline
\end{tabular}


a)

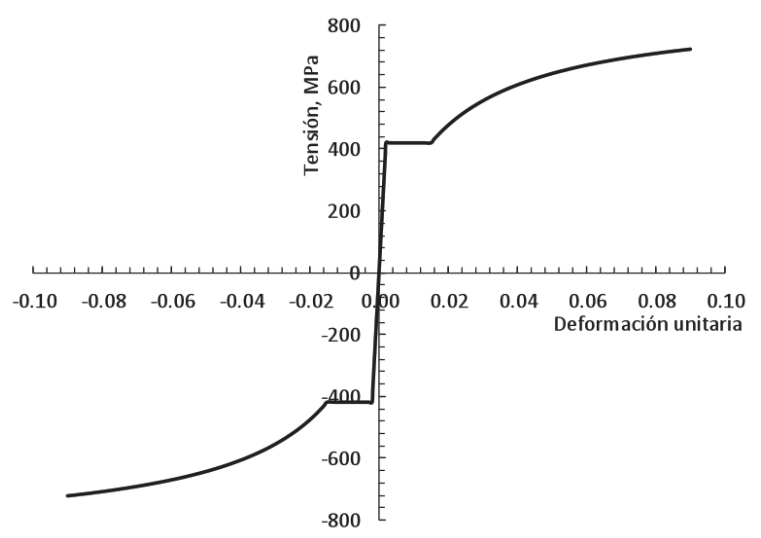

b)

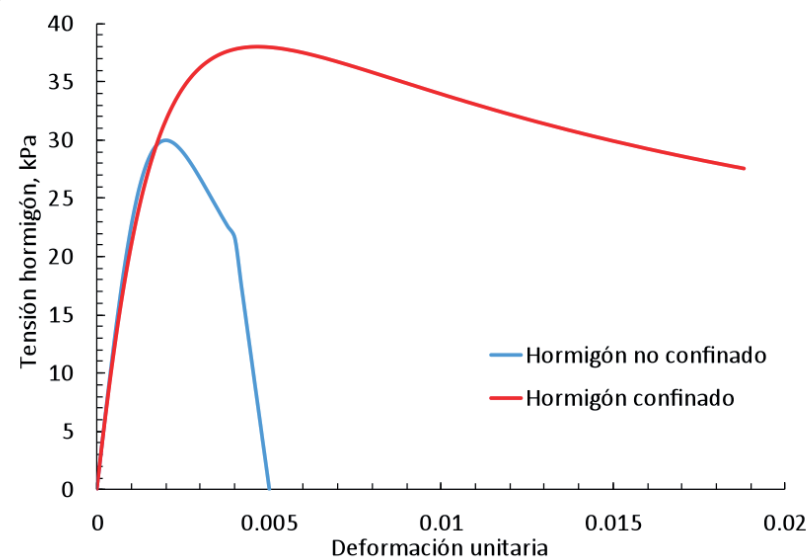

Figura 5: Curvas de tensión-deformación: a) modelo de Park para acero de refuerzo A630-420H y b) modelo de Mander para hormigón confinado y no confinado

patrón triangular invertido presentan valores similares, en donde hay un incremento a medida que aumentan los pisos, en cambio el patrón masas se observa un decrecimiento a medida que aumentan los pisos.

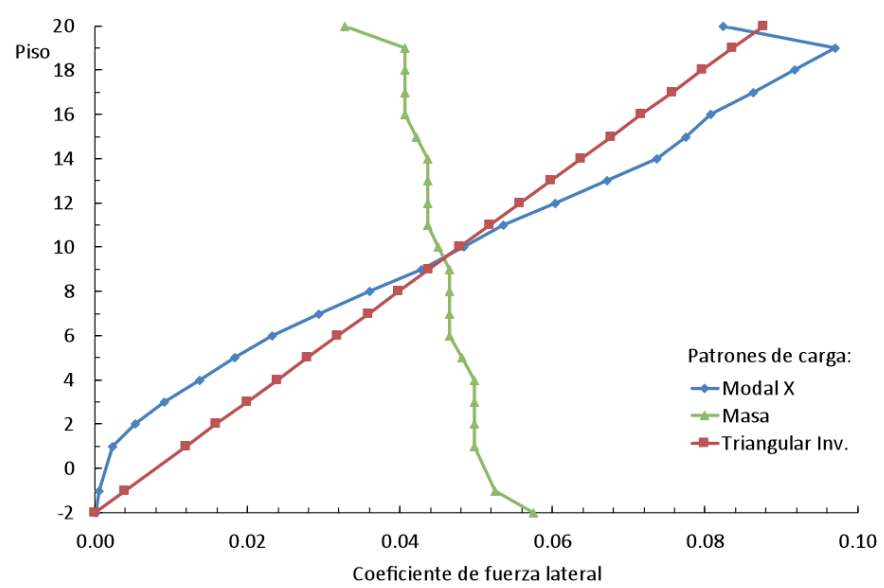

Figura 6: Comparación de coeficientes de fuerza lateral según patrones de carga

\section{Curvas de capacidad obtenidas}

Definido el modelo y los patrones de carga, se realiza el análisis pushover a través de ETABS. Del análisis se obtienen 9 curvas que relacionan el corte basal con el desplazamiento de techo. El análisis fue realizado para 3 direcciones: $+X,+Y,-Y$. No se muestra la curva de capacidad para la dirección $-X$ ya que resulta similar que para la dirección $+X$ por simetría de la estructura. En la Figura 7, se muestran las curvas de capacidad obtenidas en las 3 direcciones de análisis según los 3 patrones de carga utilizados.

a)

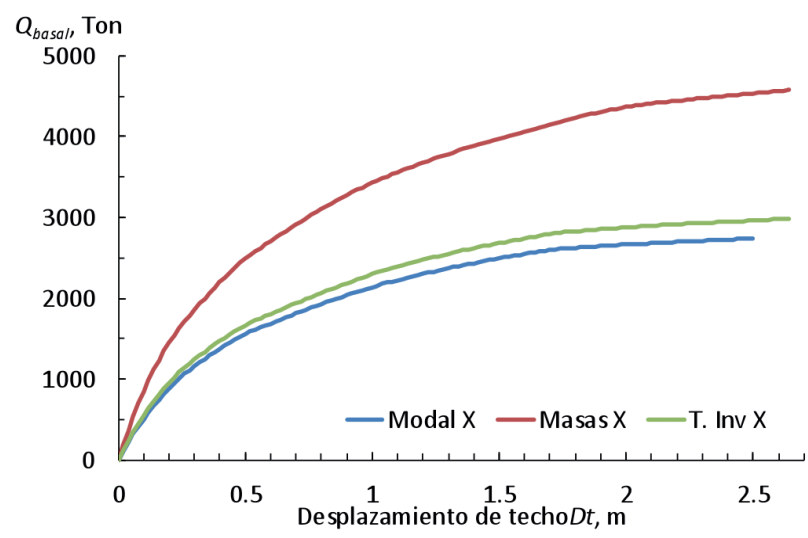

b)

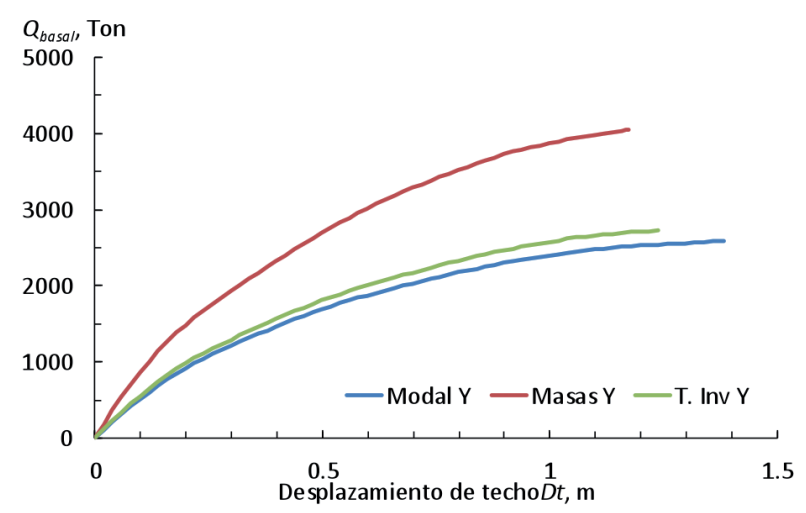

c)

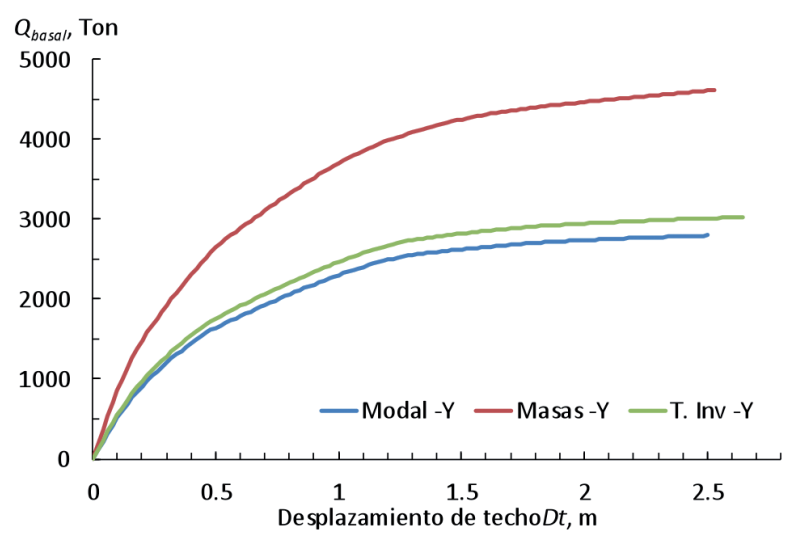

Figura 7: Curvas de capacidad obtenidas en la dirección: a) $X$, b) $+Y$ y c) $-Y$ 


\section{Demanda sísmica y desempeño}

En el marco conceptual de la ingeniería sísmica basada en el desempeño estructural, existen tres conceptos importantes que se tienen que tomar en cuenta: niveles de desempeño, demanda sísmica y objetivos de desempeño.

\section{Niveles de desempeño}

Los niveles de desempeño consideran principalmente los siguientes aspectos: daños físicos en los elementos estructurales y no estructurales, riesgos a los que se encuentran expuestos los ocupantes de la estructura o edificio en evaluación y funcionalidad, es decir, si los servicios básicos siguen o no operativos.

La ingeniería basada en el desempeño establece como referencia cuatro niveles de desempeño (SEAOC, 1995):

- Totalmente operacional: daño estructural y no estructural despreciable o nulo.

- Operacional: agrietamientos en elementos estructurales. Daño leve.

- Seguridad de vida: daños moderados en algunos elementos. Pérdida de resistencia y rigidez del sistema resistente de cargas laterales. El sistema permanece funcional.

- Pre-colapso: daños severos en elementos estructurales. Puede llegar a ser necesario demoler el edificio.

\section{Demanda sísmica}

Vision 2000 (SEAOC, 1995) especifica cuatro niveles de demanda definidos según su probabilidad de ocurrencia y período de retorno. Ellos son definidos como: movimientos sísmicos de diseño frecuente, ocasional, raro y muy raro. Sin embargo, estos sismos están orientados a la realidad geosísmica norteamericana, por lo que requieren necesariamente de una calibración de sus límites adecuándose a la realidad sismotectónica chilena. Lagos et al. (2012) definen que cada nivel de demanda queda representado por espectros elásticos (aceleración elástica espectral $S_{a}$ ), los cuales están en función de parámetros definidos en la norma sísmica NCh433 (2010) y el DS61 (2011). En la Tabla $3, f_{\min }=Q_{\min } / Q_{\text {basal }}$ si $Q_{\text {basal }}<Q_{\text {mín }}$.

Para esta investigación se consideran los 4 niveles de demanda sísmica, además de 2 formas para determinar espectros de desplazamiento elástico: el primero señalado por FEMA 440 (2005) y utilizado en Chile antes del DS61 (2011) y el segundo según lo establecido por el DS61 (2011). En la Figura 8 se comparan espectros de demanda según las dos formas consideradas para determinar el desplazamiento elástico, para sismo frecuente y sismo muy raro.

Tabla 3: Niveles de demanda sísmica

\begin{tabular}{|l|c|c|c|}
\hline $\begin{array}{c}\text { Movimiento } \\
\text { sísmico de } \\
\text { diseño }\end{array}$ & $\begin{array}{c}\text { Periodo de } \\
\text { retorno, años }\end{array}$ & $\begin{array}{c}\text { Probabilidad de } \\
\text { excedencia, \% }\end{array}$ & $\begin{array}{c}\text { Nivel de } \\
\text { demanda } \\
\text { sísmica }\end{array}$ \\
\hline Frecuente & 43 & 50 en 30 años & $S_{a}=\frac{f_{\min } S A_{0} \alpha}{\left(R^{*} / I\right)}$ \\
\hline Ocasional & 72 & 50 en 50 años & $S_{a}=1.4 \frac{f_{\min } S A_{0} \alpha}{\left(R^{*} / I\right)}$ \\
\hline Raro & 475 & 10 en 50 años & $S_{a}=S A_{0} \alpha I$ \\
\hline Muy raro & 970 & 10 en 100 años & $S_{a}=1.2 S A_{0} \alpha I$ \\
\hline
\end{tabular}

a)

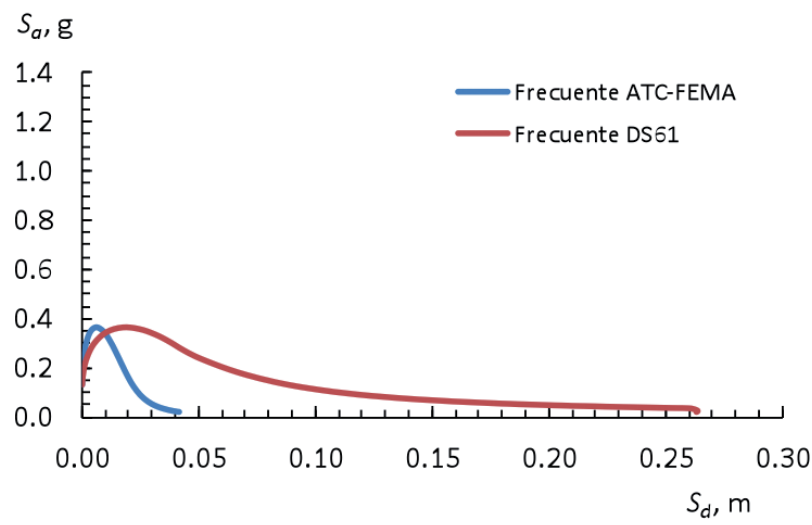

b) $S_{a}, g$

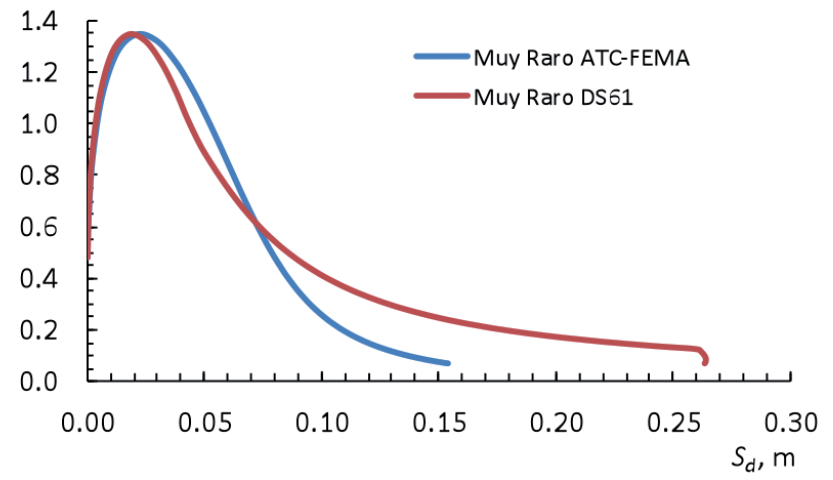

Figura 8: Comparación de espectros de demanda: a) sismo frecuente y b) sismo muy raro

\section{Objetivos de desempeño}

Se definen como una expresión del grado deseado de daño (nivel de desempeño) ante una intensidad de sismo (nivel de demanda sísmica). Vision 2000 propone objetivos múltiples mínimos de desempeño, en lo que denomina 
matriz de objetivos de diseño (Tabla 4), para diferentes tipos de estructuras clasificadas según sus implicancias en la seguridad poblacional.

Tabla 4: Matriz de objetivos de diseño - Vision 2000 (SEAOC, 1995)

\begin{tabular}{|c|c|c|c|c|}
\hline \multirow{2}{*}{$\begin{array}{l}\text { Nivel de } \\
\text { demanda } \\
\text { sísmica }\end{array}$} & \multicolumn{4}{|c|}{ Nivel de desempeño sísmico } \\
\hline & $\begin{array}{c}\text { Totalmente } \\
\text { operacional }\end{array}$ & Operacional & $\begin{array}{c}\text { Seguridad de } \\
\text { vida }\end{array}$ & $\begin{array}{c}\text { Pre } \\
\text { colapso }\end{array}$ \\
\hline Frecuente & $\mathrm{OB}$ & $\mathrm{X}$ & $\mathrm{X}$ & $\mathrm{X}$ \\
\hline Ocasional & OEP & OB & $\mathrm{X}$ & $\mathrm{X}$ \\
\hline Raro & OSC & OEP & OB & $\mathrm{X}$ \\
\hline Muy raro & - & OSC & OEP & OB \\
\hline
\end{tabular}

OB: Objetivo Básico: edificio habitacional y de oficinas, X: objetivo inaceptable

OEP: Objetivo Esencial y Peligroso: edificios esenciales (hospital, policía) e instalaciones peligrosas

OSC: Objetivo de Seguridad Crítica: edificios con elementos tóxicos, explosivos, radiactivos, etc.

En la Tabla 5 se muestran los desplazamientos objetivos para cada uno de los niveles de desempeño propuestos por Vision 2000, los cuales representan la relación $D t / H$, en que $D t$ es el desplazamiento lateral máximo del nivel superior del edificio con respecto a la base (desplazamiento de techo) y $H$ es la altura del edificio. Para cumplir con los objetivos de desempeño, los desplazamientos objetivos deben ser inferiores a cada uno de estos valores señalados en la Tabla 5.

Tabla 5: Valores de desplazamiento objetivo máximo para niveles de desempeño

\begin{tabular}{|l|c|}
\hline \multicolumn{1}{|c|}{ Nivel de desempeño } & $\begin{array}{c}\text { Desplazamiento objetivo } \mathrm{Dt} / \mathrm{H}, \\
\%\end{array}$ \\
\hline Totalmente Operacional & $\leq 0.2$ \\
\hline Operacional & $\leq 0.5$ \\
\hline Seguridad de Vida & $\leq 1.5$ \\
\hline Pre Colapso & $\leq 2.5$ \\
\hline Colapso & $>2.5$ \\
\hline
\end{tabular}

\section{Método de espectro de capacidad MEC}

El método de espectro de capacidad es un procedimiento ampliamente utilizado para determinar puntos de desempeño. Dicho método mediante un procedimiento gráfico, compara la capacidad de la estructura para resistir fuerzas laterales con la demanda sísmica, representada por medio de un espectro elástico reducido. El método está expuesto en el ATC-40 (1996), sin embargo, FEMA 440 (2005) introduce modificaciones que han sido consideradas en esta investigación.

\section{Procedimiento para obtener puntos de desempeño a través del MEC}

1. Obtener la curva de capacidad mediante análisis estático no lineal pushover.

2. Transformar la curva de capacidad a formato ADRS (coordenadas espectrales $S_{a}$ y $S_{d}$ ), utilizando factores dinámicos de la estructura: el factor de participación modal $P F_{R}$ y el coeficiente de masa modal efectiva $\alpha_{R}$.

$$
\begin{aligned}
& P F_{R}=\frac{\sum_{i=1}^{N} m_{i} \phi_{i R}}{\sum_{i=1}^{N} m_{i} \phi_{i R}{ }^{2}} \\
& \alpha_{R}=\frac{\left[\sum_{i=1}^{N} m_{i} \phi_{i R}\right]^{2}}{\sum_{i=1}^{N} m_{i} \sum_{i=1}^{N} m_{i} \phi_{i R}{ }^{2}} \\
& S_{d}=\frac{D t}{P F_{R}} \\
& S_{a}=\frac{Q_{\text {basal }}}{\alpha_{R} M} \\
& M=\sum_{i=1}^{N} m_{i}
\end{aligned}
$$

3. Transformar la curva de capacidad a una curva de capacidad bilineal, esto para obtener una representación idealizada en donde se define un punto de fluencia A de coordenadas $a_{y}-d_{y}$ y un punto de desempeño supuesto B de coordenadas $a_{p i}-d_{p i}$, como se muestra en la Figura 9. Para validar dicha curva, el error entre el área de la curva original y el área de la curva bilineal tiene que ser menor al 5\%.

4. Determinar los parámetros de linealización equivalente propuestos por FEMA 440 (2005) correspondientes a: periodo efectivo $T_{\text {eff }}$ y amortiguamiento efectivo $\beta_{\text {eff }}$ Estos son valores optimizados para cualquier curva de capacidad, independiente de su ciclo histerético o rigidez post-elástica $\alpha$. Estos parámetros lineales efectivos están en función de características de la curva de capacidad (CS: Capacity Spectrum), tales como: periodo inicial $T_{0}$, amortiguamiento inicial $\beta_{0}$ y ductilidad $\mu$. En esta investigación se muestran las ecuaciones para el tramo de ductilidad menor a $4(1<$ $\mu<4)$. 
$\beta_{\text {eff }}=4.9(\mu-1)^{2}-1.1(\mu-1)^{3}+\beta_{0}$

$T_{\text {eff }}=\left[0.2(\mu-1)^{2}-0.038(\mu-1)^{3}+1\right] T_{0}$

5. Determinar el factor de reducción $B\left(\beta_{\text {eff }}\right)$ de la curva de demanda ADRS $\left(\beta_{0}\right)$ según la Figura 10. Este factor reduce coordenadas de aceleración espectral, reduciendo la aceleración espectral inicial $\left(S_{a}\right)_{0}$ a una aceleración espectral efectiva $\left(S_{a}\right)_{\beta}$, en función del amortiguamiento efectivo $\beta_{\text {eff }}$ obteniéndose la curva de demanda reducida $\operatorname{ADRS}\left(\beta_{\text {eff }}\right)$.

$$
\begin{aligned}
& \left(S_{a}\right)_{\beta}=\frac{\left(S_{a}\right)_{0}}{B\left(\beta_{e f f}\right)} \\
& B\left(\beta_{e f f}\right)=\frac{4}{5.6-\ln \left(\beta_{e f f}\right)}
\end{aligned}
$$

6. Determinar el factor de modificación $M$, que relaciona el periodo secante $T_{s e c}$ y el periodo efectivo $T_{\text {eff }}$, modificando la curva de demanda reducida ADRS $\left(\beta_{\text {eff }}\right)$ (Figura 10). Este factor transforma coordenadas de aceleración espectral $a_{\text {eff }}$, en aceleración máxima $a_{\max }$, obteniéndose la curva de demanda modificada MADRS $\left(\beta_{\text {eff }}, M\right)$.

$$
\begin{aligned}
& M=\frac{a_{\text {max }}}{a_{e f f}} \rightarrow a_{\text {max }}=a_{e f f} M \\
& M=\left[\frac{T_{e f f}}{T_{s e c}}\right]=\left[\frac{T_{e f f}}{T_{0}}\right]^{2}\left[\frac{T_{0}}{T_{s e c}}\right]^{2} \\
& {\left[\frac{T_{0}}{T_{s e c}}\right]^{2}=\frac{1+\alpha(\mu-1)}{\mu}} \\
& \alpha=\frac{\left(\frac{a_{p i}-a_{y}}{d_{p i}-d_{y}}\right)}{\left(\frac{a_{y}}{d_{y}}\right)} \\
& \mu=\frac{d_{p i}}{d_{y}}
\end{aligned}
$$

Donde $\mu$ es la ductilidad y $\alpha$ es la rigidez post-elástica.

7. Obtener un punto de desempeño de la intersección de la curva de capacidad bilineal y la curva de demanda modificada MADRS $\left(\beta_{e f f}, M\right)$.
8. Transformar punto de desempeño de valores espectrales $\left(S_{a}, S_{d}\right)$ a valores de desplazamiento de techo $D t$ y corte basal $Q_{\text {basal }}$, utilizando los factores dinámicos de la estructura calculados en el punto 2 .

$$
\begin{aligned}
& D t=S_{d} P F_{R} \\
& Q_{\text {basal }}=S_{a} \alpha_{R} M \\
& M=\sum_{i=1}^{N} m_{i}
\end{aligned}
$$

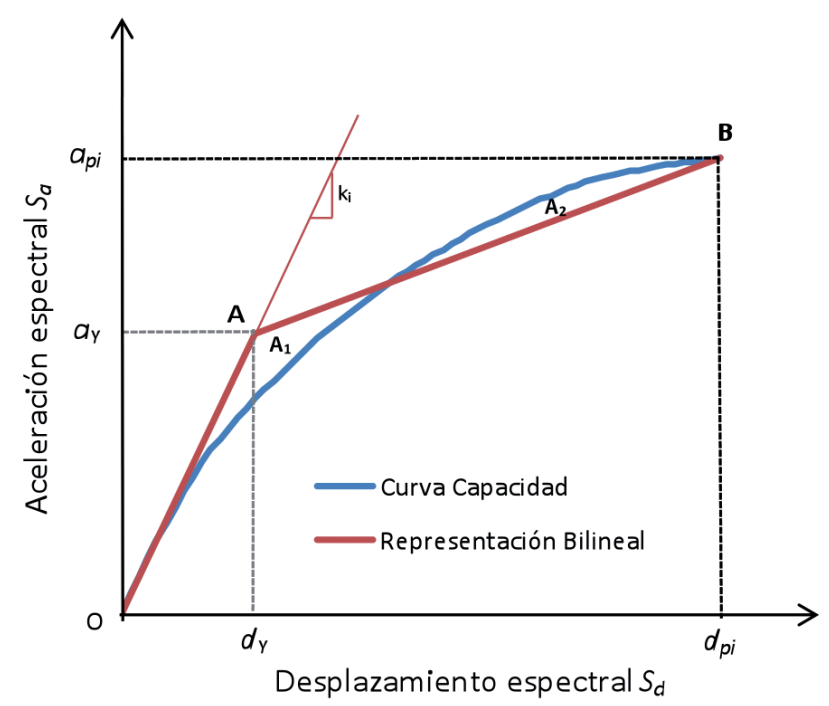

Figura 9: Representación bilineal de la curva de capacidad

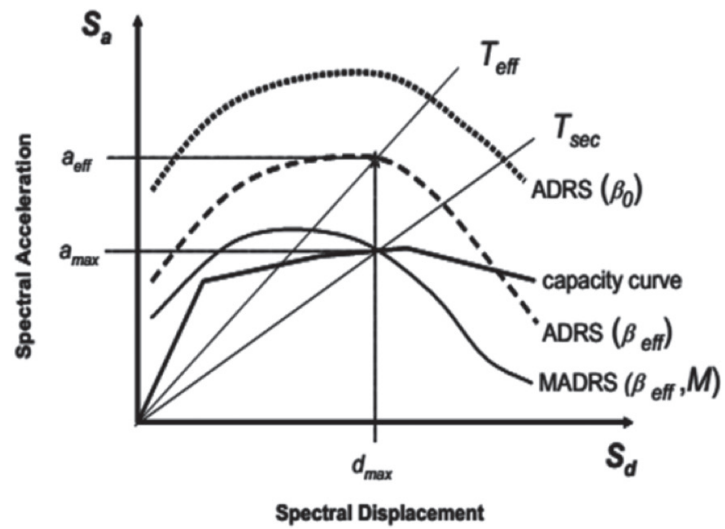

Figura 10: Demanda reducida por factor $B\left(\beta_{\text {eff }}\right)$ y modificada por factor $M$ (FEMA 440, 2005) 


\section{Resultados}

\section{Puntos de desempeño}

En las Figuras 11 y 12 se muestran los resultados de puntos de desempeño (desplazamiento de techo $D t$ y corte basal $Q)$ considerando los 3 patrones de carga, los 4 niveles de demanda y 2 espectros de desplazamiento elástico antes señalados. Se muestran los resultados para dirección de análisis $+X \mathrm{e}-Y$ que resultaron ser las más desfavorables.

a)
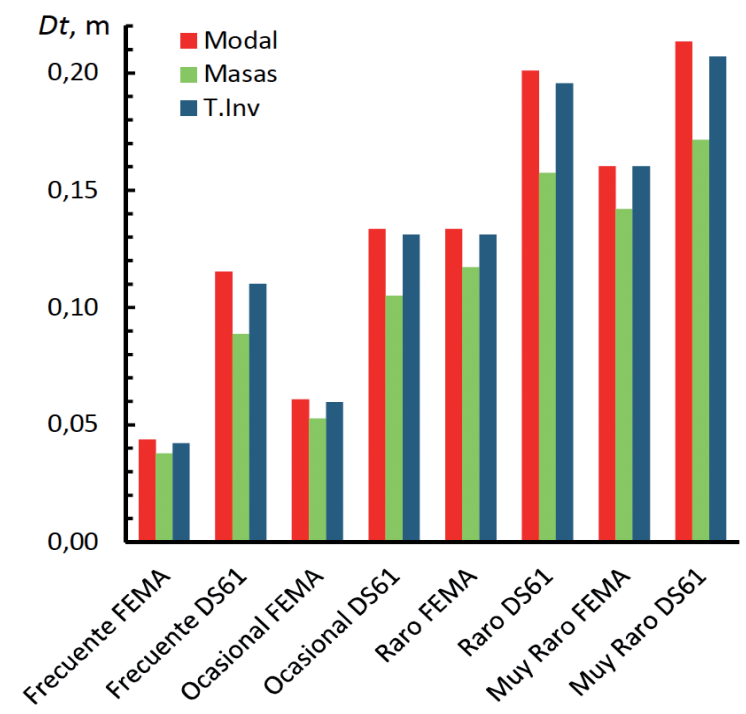

b)

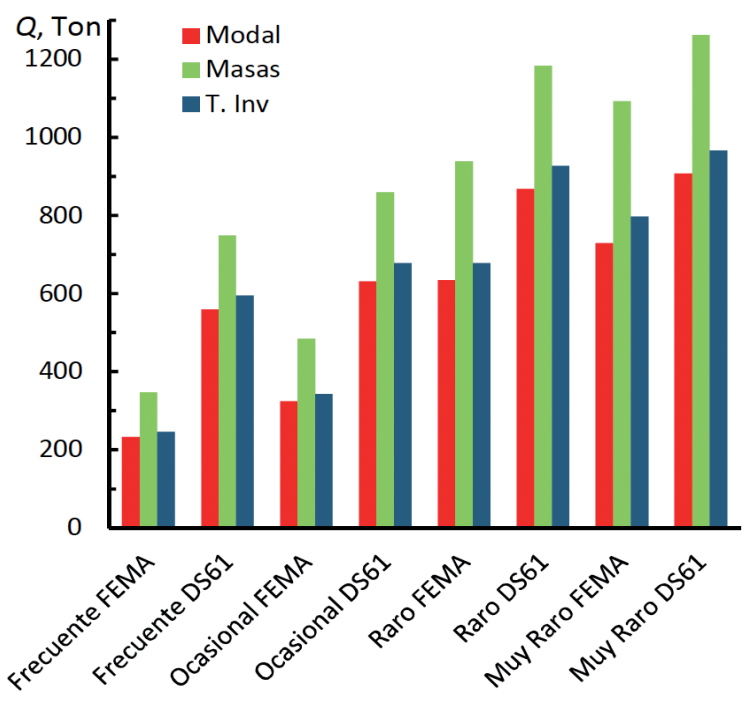

Figura 11: Puntos de desempeño en la dirección $+X$, a) desplazamiento de techo y b) corte basal

Al comparar diferencias de puntos de desempeño entre las dos formas consideradas para determinar espectros de desplazamiento elástico, se observan grandes diferencias en niveles de demanda sísmica frecuente, hasta $167 \%$ en desplazamiento techo y $165 \%$ en corte basal y ocasional, hasta $125 \%$ en desplazamiento de techo y $118 \%$ en corte basal. En tanto para sismo raro y muy raro alcanzan diferencias hasta de un $50 \%$ y $29 \%$ en desplazamiento de techo y hasta un $37 \%$ y $21 \%$ en corte basal al aplicar DS 61 (2011) con respecto a FEMA 440 (2005).

a)

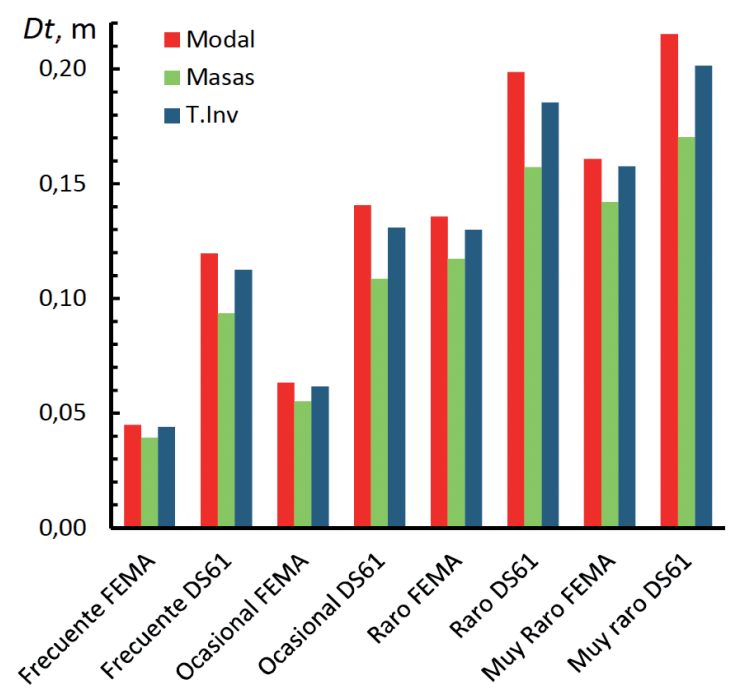

b)

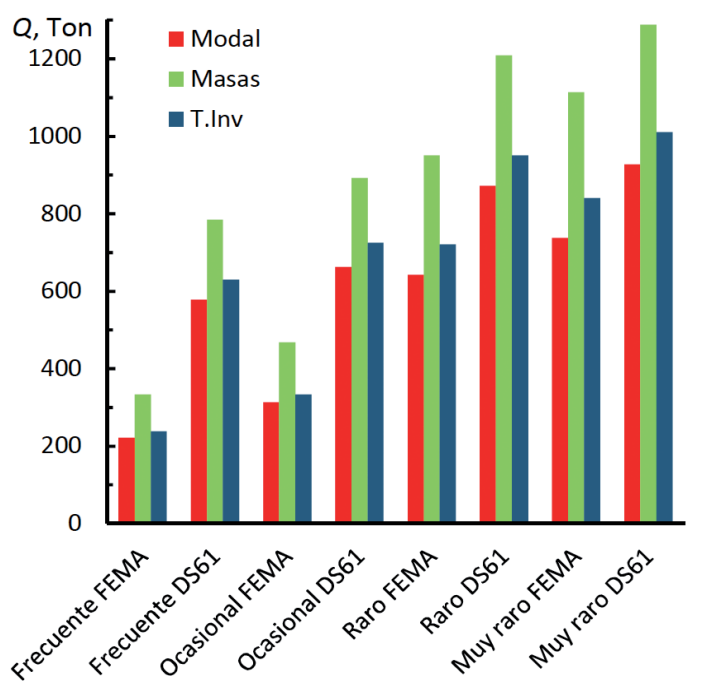

Figura 12: Puntos de desempeño en la dirección $-Y$, a) desplazamiento de techo y b) corte basal

Con respecto a patrones de carga utilizados, se observan diferencias mayores entre patrón modal y masas, en donde el patrón de masas origina hasta un $50 \%$ más de corte basal que el patrón modal y hasta un $25 \%$ menos en desplazamiento de techo.

Al comparar resultados entre direcciones de análisis, las mayores diferencias se encuentran entre dirección $-Y$ con 
respecto a dirección $+X$, las que alcanzan valores de hasta un $7.2 \%$ mayor en desplazamiento de techo y un $11.6 \%$ menor en corte basal.

\section{Desplazamiento objetivo}

Tal como se señaló anteriormente, Vision2000 propone ciertos límites en donde el desplazamiento objetivo $D t / H$, no puede superar cierto porcentaje según el nivel de desempeño que se analiza (Tabla 5). En la Figura 13 se muestran los desplazamientos objetivos $D t / H$ y se comparan con los límites de Vision 2000. Se observa que todos los puntos de desempeño no superan los límites establecidos por Vision 2000, independiente del patrón de carga, nivel de demanda sísmica, forma para determinar el espectro de desplazamiento elástico o dirección de análisis seleccionada, por lo tanto, se cumple con los criterios de Vision 2000. Cabe destacar que en sismos frecuentes no se supera el límite totalmente operacional TO, en tanto en las demás demandas sísmicas no se supera el límite operacional OP. Por lo que el edificio mantiene un nivel de desempeño operacional ante sismos de gran intensidad.

\section{Desplazamiento relativo entrepisos medido en el centro de masa}

La actual normativa sísmica chilena considera un desplazamiento relativo máximo entre dos pisos consecutivos, medido en el centro de masa para cada una de las direcciones analizadas, que no debe ser mayor que la altura de entrepiso multiplicada por 0.002 , es decir, un drift entrepisos que no debe superar un $0.2 \%$, como condición para controlar desplazamientos por traslación (rigidez traslacional del edificio). Dicha consideración es equivalente al límite de Vision 2000 totalmente operacional TO asociado a una demanda sísmica frecuente.

En las Figuras 14 y 15 se comparan los drift entrepisos en dirección $-Y$ (que en esta investigacion resulta ser la dirección de análisis más desfavorable) con el drift entrepisos resultante del análisis sísmico convencional requerido por la normativa chilena denominado sismo de diseño.

En la Figura 14 se observa que en sismo frecuente FEMA 440 (2005) y en sismo frecuente DS61 (2011) se obtienen valores de drift entrepisos superiores a los presentados a) $D t / H, \%=$ Modal $=$ Masas $=T . I n v-$ Totalmente Operacional — Operacional

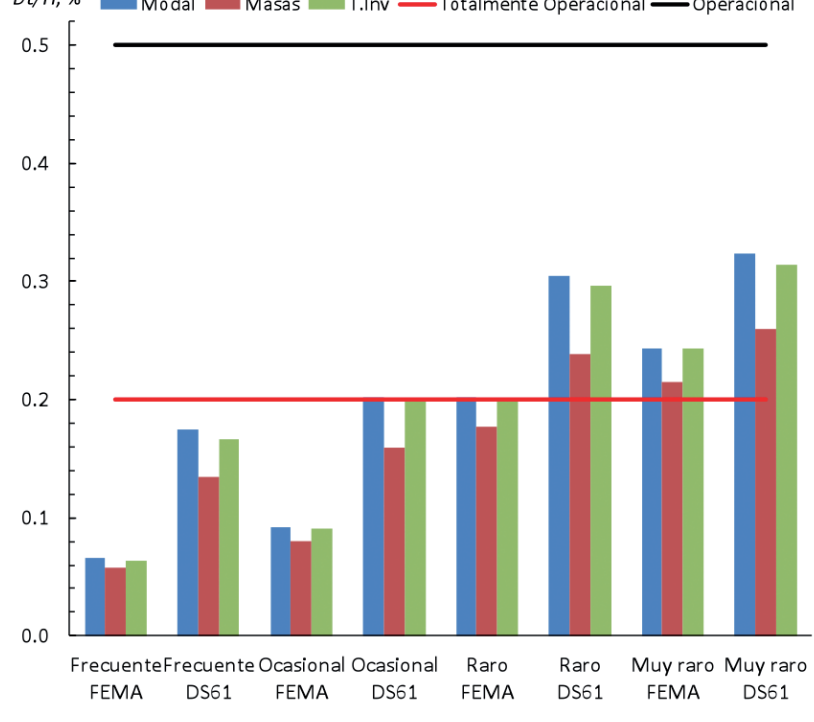

$D t / H, \%$ Modal $=$ Masas $=$ T.Inv — Totalmente Operacional —operaciona

b)

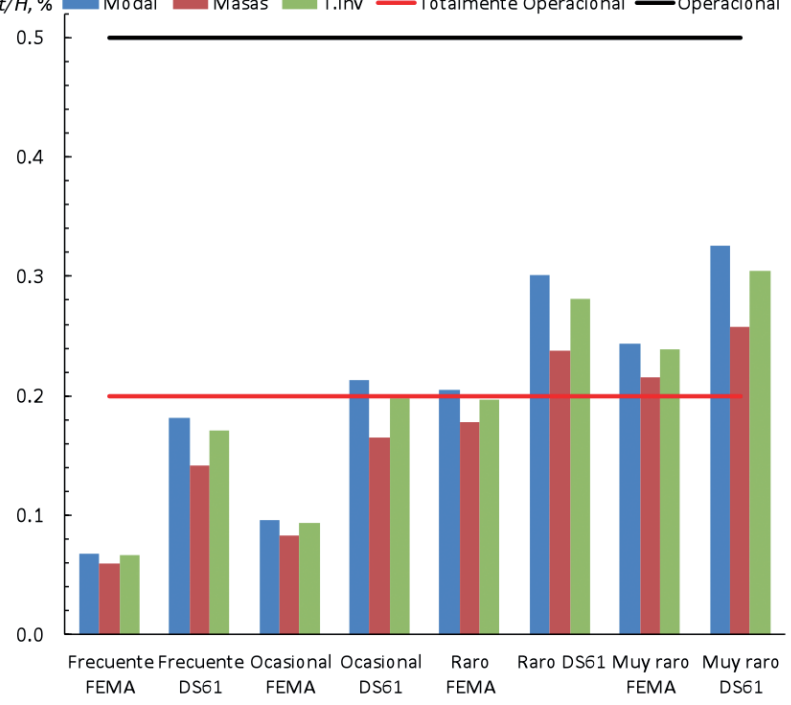

Figura 13: Desplazamiento objetivo versus límites de Vision 2000 en la dirección: a) $+X$ y b) $-Y$

por el análisis sísmico de la normativa chilena (sismo de diseño). En tanto en sismo frecuente DS61 (2011) se supera el límite establecido por Vision 2000 en patrones modal y triangular invertido.

En la Figura 15 se observa que, en los casos más desfavorables, es decir, sismo raro y muy raro (ambos con espectro de desplazamiento elástico establecido por el DS61), los drift de entrepiso nunca superan el límite operacional $(0.5 \%)$. 
a)

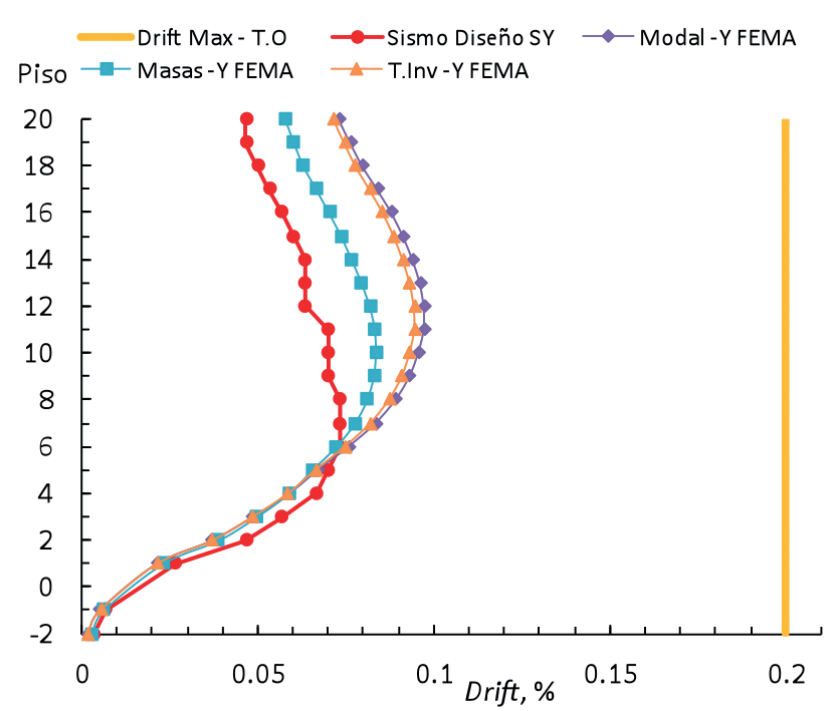

b)

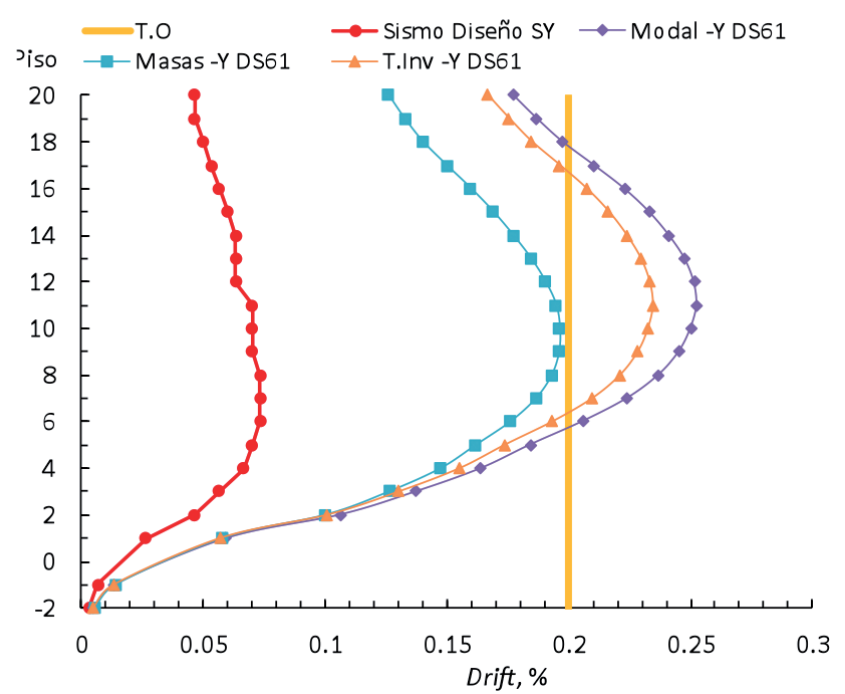

Figura 14: Comparación drift entrepisos, sismo de diseño versus: a) sismo frecuente FEMA 440 (2005) y b) sismo frecuente DS $61(2011)$

\section{Cortes por piso}

En la Figura 16 se muestran los cortes por piso de 2 niveles de demanda sísmica comparados con los resultados obtenidos del análisis sísmico realizado según requerimientos de normativa chilena, sismo de diseño (sismo $Y$ ). Para el nivel de demanda sísmica, sismo frecuente DS61 (Figura 16a), se obtienen resultados superiores al sismo de diseño de la normativa chilena, mientras que para sismo frecuente FEMA y ocasional FEMA (Figura 16b), se obtienen resultados inferiores al sismo de diseño. Cabe destacar que se muestran cortes por piso de los 3 patrones de carga en dirección $+Y$, dirección que presentó valores más desfavorables para este análisis. a)

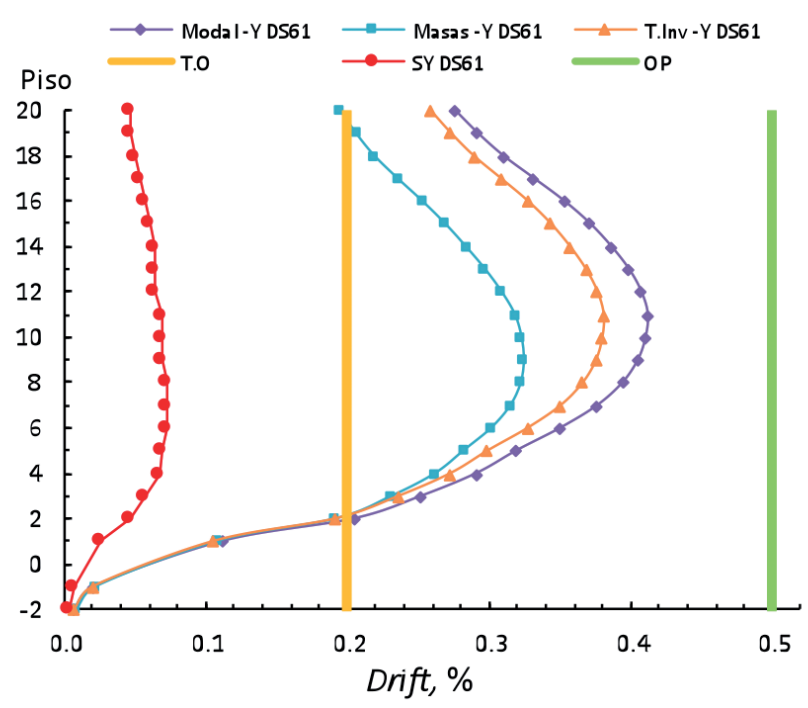

b)

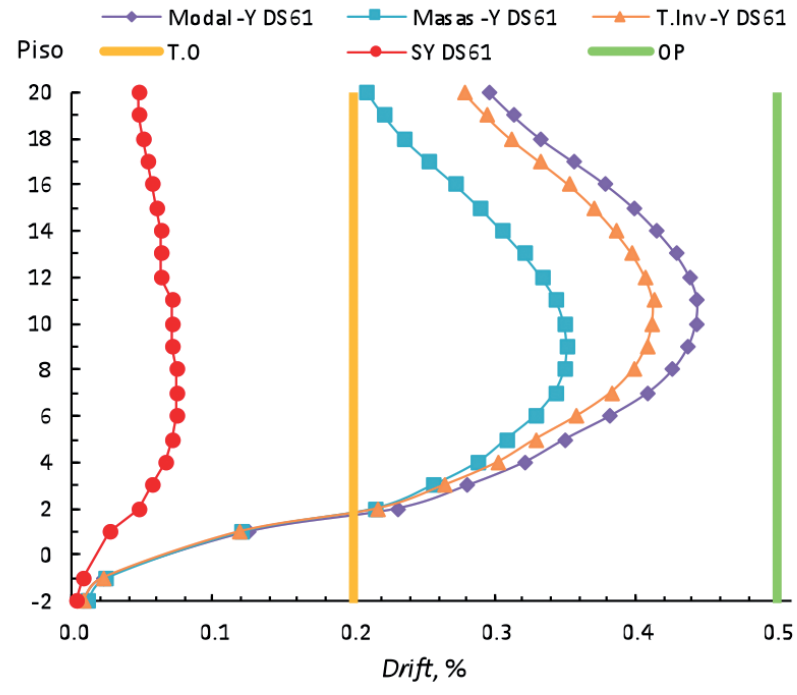

Figura 15: Comparación drift entrepisos, sismo de diseño versus: a) sismo raro DS61 (2011) y b) sismo muy raro DS61 (2011)

\section{Tensiones máximas en muros}

Se analizaron las tensiones máximas resultantes en muros para 2 casos: las obtenidas a partir de los puntos de desempeño para la demanda sísmica más desfavorable y para los desplazamientos máximos obtenidos en las curvas de capacidad. En el primer caso para los patrones de carga más desfavorables se observa en la Figura 17 que el muro $1 \mathrm{~L}$ presenta las mayores tensiones de compresión para un sismo en la dirección $X$ aplicando un patrón triangular invertido y el muro 2I para un sismo en la dirección $-Y$ aplicando un patrón modal. La dirección $+Y$ alcanza tensiones menos desfavorables que en la dirección $-Y$, por lo que no será mostrada. En el segundo caso (Figura 18) 
al aplicar un pushover hasta alcanzar un desplazamiento máximo de techo, es decir, la máxima capacidad presentada en las curvas de capacidad, se observa que, aplicando los mismos patrones de carga del primer caso, se obtienen tensiones superiores de hasta un $84.4 \%$ en la dirección $X$ y un $126.3 \%$ en dirección $-Y$ a las obtenidas con respecto a las tensiones en puntos de desempeño con la demanda sísmica más desfavorable (sismo muy raro). Además, se destaca que el muro 4L es ahora el muro con mayores tensiones, en la dirección $X$.

a)

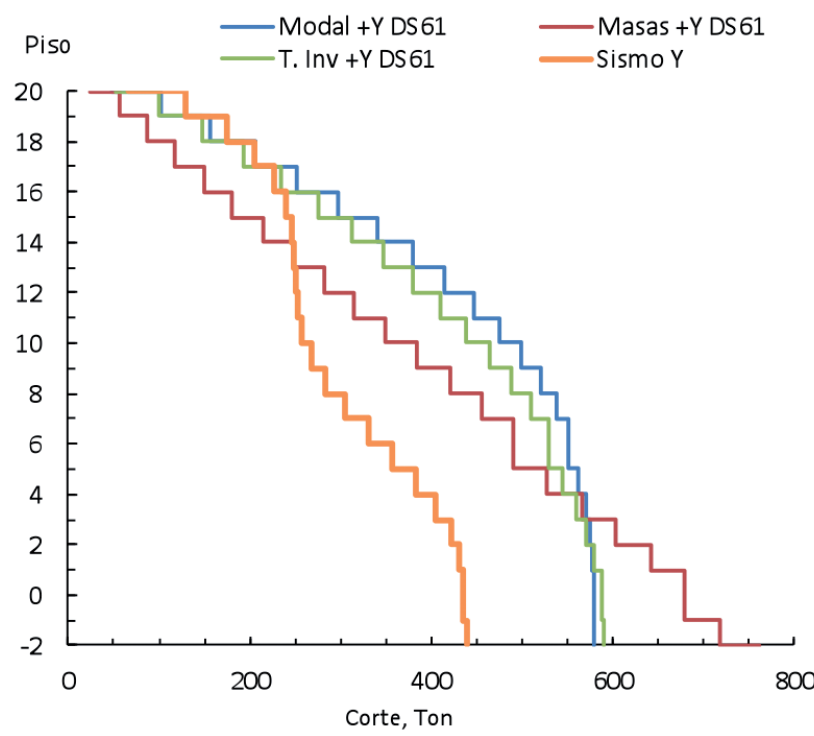

b)

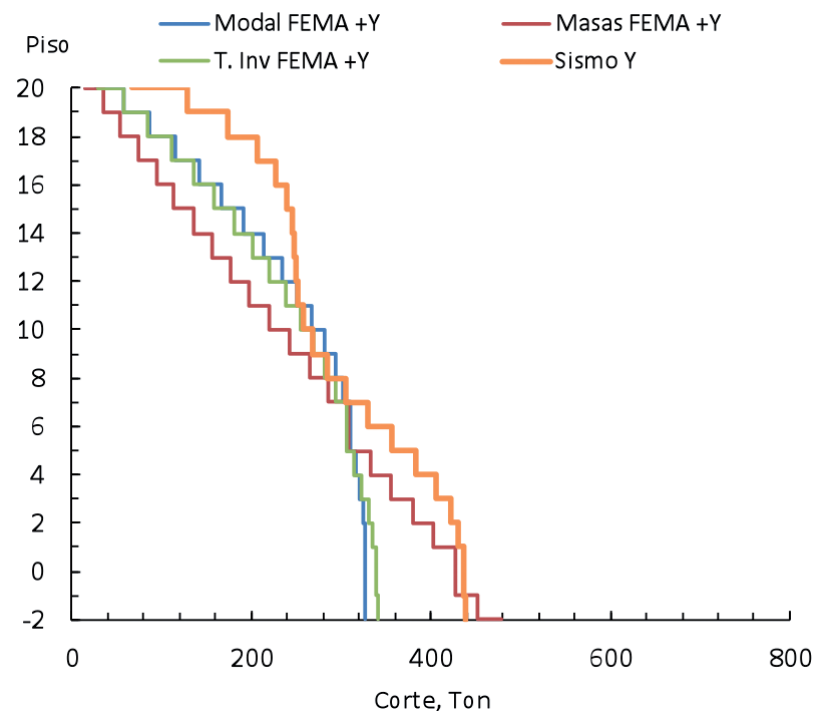

Figura 16: Cortes por piso, sismo diseño versus: a) sismo frecuente DS61 (2011) y b) sismo ocasional FEMA 440 (2005) a)

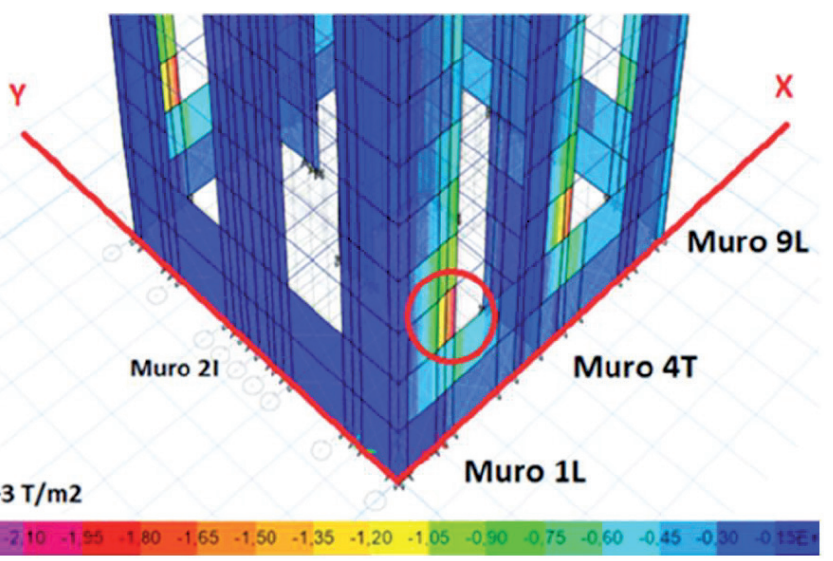

b)

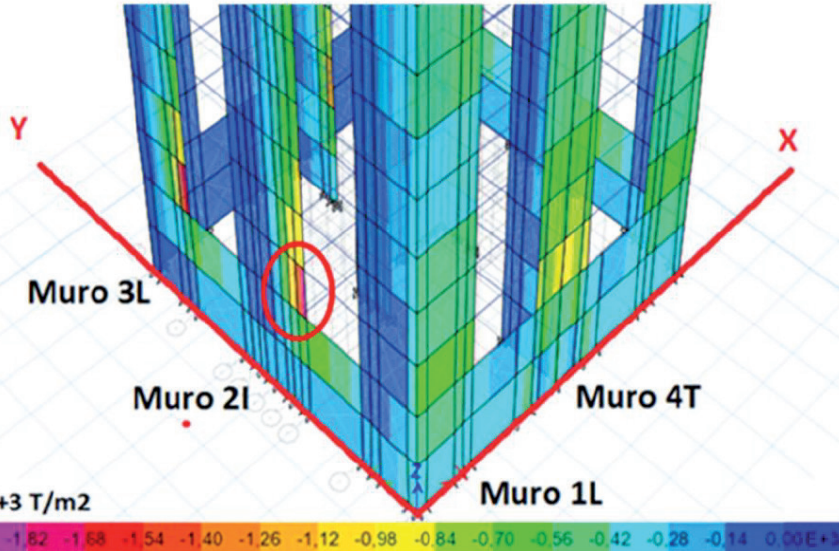

Figura 17: Tensión máxima (círculo rojo): a) $2156 \mathrm{~T} / \mathrm{m}^{2}$, muro 1L, piso 1, sismo muy raro DS61 (2011), patrón T. inv. $X$ y b) $1946 \mathrm{~T} / \mathrm{m}^{2}$, muro 2I, piso 1, sismo muy raro DS61 (2011), patrón modal $-Y$.

\section{Conclusiones}

1. Todos los puntos de desempeño no superan los límites establecidos por Vision 2000, independiente del patrón de carga, nivel de demanda sísmica, forma de determinar el espectro de desplazamiento elástico o dirección de análisis considerada. Por lo tanto, el edificio cumple con los criterios de Vision 2000.

2. El desempeño alcanzado por el edificio estudiado para sismos frecuentes es totalmente operacional. Para los demás niveles de demanda sísmica el desempeño alcanzado es operacional. Por lo tanto, el edificio mantiene un nivel de desempeño operacional ante sismos de gran intensidad. Este nivel de desempeño coincide con la experiencia chilena, según Lagos et al. (2012). 
a)

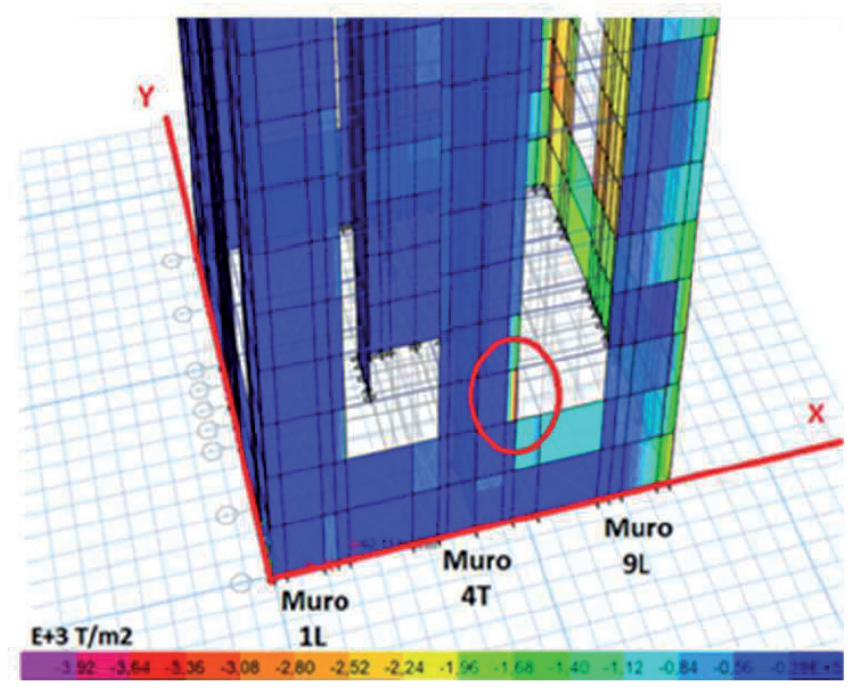

b)

\section{$E+3 T / m 2$}

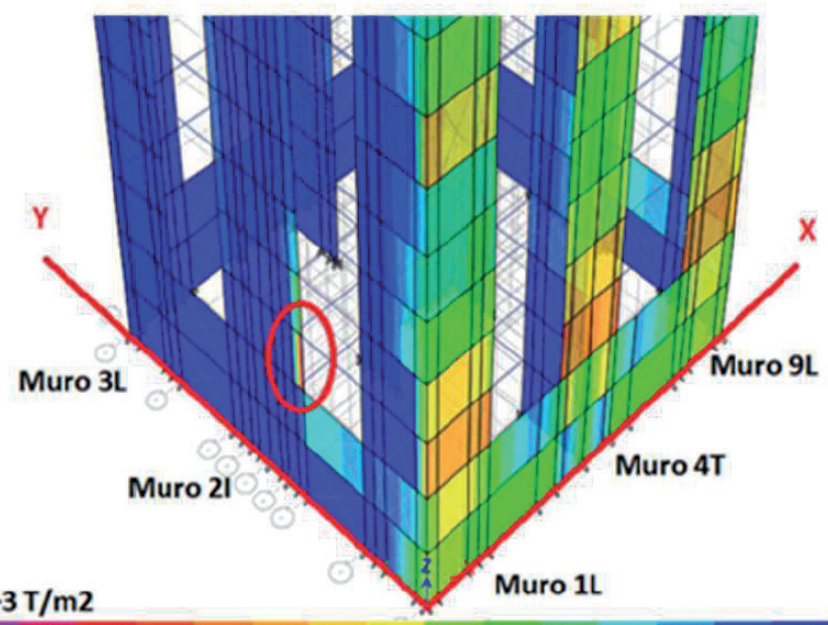

Figura 18: Capacidad máxima (círculo rojo): a) 3975 T/m², muro 4T, piso 1, patrón T. inv. $X$ y b) $4404 \mathrm{~T} / \mathrm{m}^{2}$, muro 2I, piso 1, patrón modal $-Y$

3. En relación a la forma de determinar el espectro de desplazamiento elástico, se encuentran importantes diferencias en los valores que se obtienen para sismo frecuente y ocasional. Es así, que producto de ello resultan diferencias en los desplazamientos de techo de hasta un $167 \%$ para sismos frecuentes y $125 \%$ para sismos ocasionales y en el caso de corte basal de hasta un $165 \%$ para sismos frecuentes y $118 \%$ para sismos ocasionales, al aplicar DS61 (2011) con respecto a FEMA 440 (2005).

4. Respecto a los patrones de carga utilizados para determinar la curva de capacidad del edificio se concluye que, para el desplazamiento de techo el patrón modal alcanza valores de hasta un $25 \%$ mayor que el patrón de masas y que para el corte basal el patrón de masas alcanza valores de hasta un 50\% mayor que el patrón modal.

5. Respecto a la comparación de los drift entrepisos obtenidos de los puntos de desempeño evaluados versus los drift entrepisos del análisis sísmico convencional se concluye que los primeros nunca superan el límite operacional $(0.5 \%)$. Sin embargo, la mayoría de los casos supera el límite de desplazamiento relativo máximo (drift $>0.2 \%$ ) requerido por la normativa chilena (NCh433, 2010).

6. En relación a los cortes por piso se concluye que solo para sismo frecuente y ocasional según FEMA 440 (2005) se obtienen resultados inferiores o similares al análisis sísmico realizado según los requerimientos de la normativa chilena. En el caso del desplazamiento relativo máximo se concluye que para todas las demandas consideradas sus resultados son mayores al análisis sísmico requerido por la normativa chilena.

7. Respecto a las tensiones en muros se concluye que, para los puntos de desempeño obtenidos, los muros $4 \mathrm{~T}$ y $1 \mathrm{~L}$ son los que presentan las tensiones máximas de compresión para sismos en la dirección $X$ y los muros 2I y 8 I en el caso de sismos en la dirección $Y$.

8. Al aplicar un pushover hasta alcanzar un desplazamiento máximo de techo, es decir, la máxima capacidad presentada en las curvas de capacidad, se obtienen tensiones superiores de hasta un $84.4 \%$ en la dirección $X$ y un $126.3 \%$ en la dirección $-Y$ a las obtenidas con respecto a las tensiones en puntos de desempeño con la demanda sísmica más desfavorable (sismo muy raro). Se destaca que en la dirección $X$ el muro 1L tiene mayores tensiones que el muro 4T.

\section{Referencias}

ATC-40 (1996). Seismic evaluation and retrofit of concrete buildings. Applied Technology Council, Redwood City, USA

DS60 (2011). Reglamento que fija los requisitos de diseño y cálculo para el hormigón armado. Ministerio de Vivienda y Urbanismo, Chile

DS61 (2011). Reglamento que fija el diseño sísmico de edificios. Ministerio de Vivienda y Urbanismo, Chile 
ETABS (2015). Computers and Structures Inc. Analysis R Berkeley, USA

Fahjan, Y.M., Kubin, J. and Tan, M.T. (2010). Nonlinear analysis methods for reinforced concrete buildings with shear walls. $14^{\text {th }}$ European Conference on Earthquake Engineering, vol. 5, 33803387

FEMA 273 (1997). NEHRP guidelines for the seismic rehabilitation of buildings. Federal Emergency Management Agency, Washington DC, USA

FEMA 274 (1997). NEHRP commentary on the guidelines for the seismic rehabilitation of buildings. Federal Emergency Management Agency, Washington DC, USA

FEMA 356 (2000). Prestandard and commentary for the seismic rehabilitation of buildings. Federal Emergency Management Agency, Washington DC, USA

FEMA 440 (2005). Improvement of nonlinear static seismic analysis procedures. Federal Emergency Management Agency, Washington DC, USA
NCh433 (2010). Diseño sísmico de edificios. NCh433 Of.1996 mod.2009. Instituto Nacional de Normalización, Santiago, Chile NCh3171 (2010). Diseño estructural: disposiciones generales y combinaciones de cargas. NCh 3171 Of2010. Instituto Nacional de Normalización, Santiago, Chile

Lagos, R., Kupfer, M., Lindenberg, J., Bonelli, P., Saragoni, R., Gueldelman, T., Massone, L., Boroschek, R. and Yañez, F. (2012). Seismic performance of high-rise concrete buildings in Chile. International Journal of High-Rise Buildings 1(3), 181194

Mander, J.B, Priestley, M.J.N. and Park, R. (1988). Theoretical stress-strain model for confined concrete. Journal of Structural Engineering 114(8), 1804-1826

SEAOC (1995). Conceptual framework for performance based seismic engineering of buildings. Vision 2000 Committee and California Office of Emergency Services, Sacramento, USA 